\title{
Computational Modeling of a Typical Supersonic Converging-Diverging Nozzle and Validation by Real Measured Data
}

\author{
Omid Joneydi Shariatzadeh, Afshin Abrishamkar, and Aliakbar Joneidi Jafari
}

\begin{abstract}
The converging-diverging nozzles play a significant role in a supersonic wind tunnel, where they draw air from a gas reservoir. Due to the back pressure conditions through the convergent section, air reaches sonic conditions at throat. These conditions lead this stream to flow further through the divergent section where the flow Mach number increases. Manipulating the determinative variables such as area ratio and back pressure, the obtained Mach number may be regulated. In this work a comprehensive simulation of a flow in a typical supersonic converging-diverging nozzle has been reported. In the respective nozzle, flow suddenly contracts at a certain point and then expands after throat. All the simulation endeavors have been carried out by ANSYS FLUENT ${ }^{\circledR}$ utilizing the mesh geometries previously and precisely accomplished in GAMBIT ${ }^{\circledR}$. The simulations have been conducted in either 2D or 3D domains to provide better comparative platform. Also, the influence of the turbulence model, differentiation and computational grid to the solution has been studied. Furthermore, the numerical comparison between CFD modeling results and corresponding available measured data has been presented. The comparison analysis of the data demonstrates an accurate enough coordination between the experimental data and the simulation results, which is applied more to the 3D endeavors than 2Ds.
\end{abstract}

Index Terms-ANSYS FLUENT ${ }^{\circledR}$, computational fluid dynamics, converging-diverging nozzle, numerical validation, turbulence models.

\section{INTRODUCTION}

A nozzle is a proportionally plain device, specifically a formed tube which can lead hot and fast gases through. Aerospace shuttles basically use a fixed convergent section followed by a fixed divergent section as the configuration design of the nozzle [1].

There are different applications where nozzle is used to accelerate hot exhaust to generate thrust which works based on the Newton's $3^{\text {rd }}$ law of motion such as in Ramjets, scramjets, and rockets [2]. Nozzle design specifies the amount of thrust since for different nozzle designs, several

Manuscript received February 14, 2014; revised June 26, 2014.

O. Joneydi Shariatzadeh is with the Department of Energy Technology, Lappeenranta University of Technology, Skinnarilankatu 34, 53850 Lappeenranta, Finland (e-mail: Omid.Joneydi@lut.fi,).

A. Abrishamkar is with the Department of Chemical Technology, Lappeenranta University of Technology, Skinnarilankatu 34, 53850 Lappeenranta, Finland (e-mail: Afshin.Abrishamkar@lut.fi)

A. Joneidi Jafari is with the Department of Energy Technology, School of Technology, Aalto University, PO Box 14400, FI-00076 Espoo, Finland. He is also with the Department of Energy Technology, Lappeenranta University of Technology, Skinnarilankatu 34, 53850 Lappeenranta, Finland (e-mail: Aliakbar.Joneidi.Jafari@lut.fi, Ali.Joneidi@aalto.fi). parameters such as mass flow rate, outlet pressure and outlet velocity of the engine might be various [3], [4].

Based on the configurations, nozzles can be divided into three general types:

1) Cone nozzles which are conical and linear [5];

2) Bell nozzles which are contoured, shaped and classical converging-diverging [6];

3) Annular nozzles which are spike, aerospike, plug, expansion and expansion-deflection [7].

Each of the mentioned nozzles has advantages and disadvantages against the others and according to the configurations, each could be beneficial for different applications [8].

The nozzle configuration which is the topic of this research is converging-diverging nozzle. Converging-diverging nozzle was first used on steam turbines by a Swedish inventor called Gustaf de Laval which is now also well known as de Laval nozzle or Converging-Diverging Nozzle [9]. In a convergingdiverging nozzle, the hot exhaust leaves the combustion chamber and converges down to the minimum area, or throat, of the nozzle. The converging part is subsonic while in the throat Mach number is 1 and in the diverging part it reaches over unity. Mach number usually increases even after throat to the end while in some cases a small decrease in Mach number has been reported [10]. When the back pressure ratio is large enough, the flow within the entire device will be subsonic and isentropic. When the back pressure ratio reaches a critical value, the flow will become choked with subsonic flow in the converging section, sonic flow at the throat, and subsonic flow in the diverging section [11].

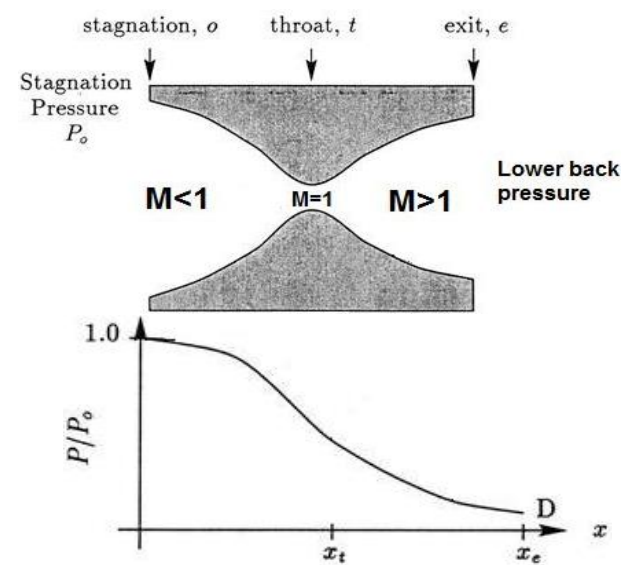

Fig. 1. Schematic view and a qualitative diagram of pressure versus the length axis for a converging-diverging nozzle with fully supersonic flow [1]

Basically, a supersonic converging-diverging nozzle has two sorts of flow trends:

1) Fully supersonic flow $(M>1)$ : As shown in Fig. 1, nozzle 
is choked; flow accelerates through the converging section, reaches its maximum speed at the throat and accelerates through the diverging section [12].

2) Shock wave (supersonic flow with shock wave): As illustrated in Fig. 2, nozzle is choked; flow accelerates through the converging section, reaches its maximum speed at the throat, accelerates through the diverging section and decelerates through the diverging section [12], [13].

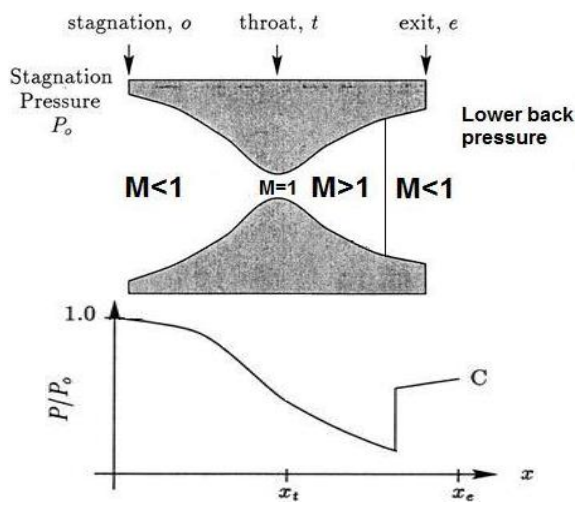

Fig. 2. Schematic view and a qualitative diagram of pressure versus the length axis for a converging-diverging nozzle with supersonic flow with shock wave.

As the matter of fact, a shock occurs after throat and the most important questions in this regard are where the shock actually happens and what the minimum pressure is after throat. To this end, there are several governing equations associated with converging-diverging nozzles that are taken into consideration in theoretical calculations, which also form the fundamentals of majority of computational fluid dynamics software such as ANSYS FLUNET ${ }^{\circledR}$, which has been applied in this work [13].

Conservation of mass [12], [14]:

$$
\rho V A=\text { constant }
$$

where $\rho=$ density $\left(\mathrm{kg} / \mathrm{m}^{3}\right), V=$ velocity $(\mathrm{m} / \mathrm{s}), A=\operatorname{area}\left(\mathrm{m}^{2}\right)$.

Conservation of momentum [12], [14]:

$$
\left(u+\frac{p}{\rho}\right)+\frac{1}{2} V^{2}=\text { constant }
$$

where $u=$ velocity magnitude $(\mathrm{m} / \mathrm{s}), p=$ pressure $\left(\mathrm{N} / \mathrm{m}^{2}(\mathrm{~Pa})\right)$. Conservation of energy [12], [15]:

$$
h+\frac{1}{2} V^{2}=\text { constant }
$$

where $h=$ enthalpy $(\mathrm{kj} / \mathrm{kg})$

In fact, the reason to use converging-diverging nozzles is to reach supersonic velocities to make the thrust even larger [16]. In converging nozzles sonic velocity is the highest velocity accessible with the extreme point at the throat, while in converging-diverging nozzles due to the increase in volume after the throat in diverging area, density drops down which causes the velocity to augment even more and reach supersonic speeds [17], [18]. Manipulating the determinative variables such as area ratio and back pressure, the obtained Mach number at the end of the nozzle may be regulated. Important applications of the converging-diverging nozzle are aerodynamics especially in jet engines where high speed aircrafts or rocket engines work [19]. They play a significant role in a supersonic wind tunnel, where they draw air from a gas reservoir which might be either at atmospheric conditions or even contains compressed and pressurized air.

In this work a comprehensive simulation of a flow in a typical supersonic converging-diverging nozzle has been reported. Simulations have been carried out by ANSYS FLUENT $^{\circledR}$ where meshing of geometries have been generated in GAMBIT $^{\circledR}$ prior to that. Two different turbulence models of $k-\varepsilon$ and $k-\omega$ have been applied to the solution. Further on, the comparison of turbulence models, grid differentiation and computational methods have been analyzed and even compared to the experimental data to give better overview of the similar applications for future purposes. The outcomes of this research demonstrate an accurate enough coordination between the experimental data and the simulation results, which is exerted more to the $3 \mathrm{D}$ endeavors than $2 \mathrm{Ds}$.

\section{GEOMETRY AND THEORY}

Converging-diverging nozzle has a non-swirling axisymmetric geometry. The 2D layout of the nozzle studied is illustrated in Fig. 3. High-pressure low velocity gas, which is air, flows through the convergent section in a subsonic condition and contracts in the throat. Then, the low-pressure high velocity air expands in divergent section in supersonic conditions [20]. Discretized method should be employed; therefore, the geometry in the shape of mesh has been developed in GAMBIT ${ }^{\circledR}$.

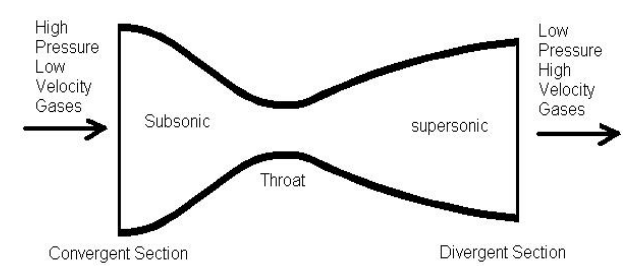

Fig. 3. 2D layout of the studied converging-diverging nozzle.

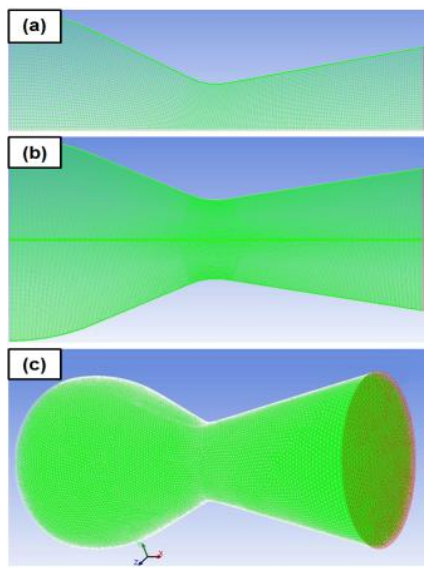

Fig. 4. Utilized geometries and meshes: a) 2D axisymmetric grid, b) 2D complete (planner) grid, and c) 3D complete grid.

In addition to the $2 \mathrm{D}$ axisymmetric, $2 \mathrm{D}$ complete (planner) case and 3D meshes were also generated to collate them in order to study the different cases. In terms of the type of mesh, the structured meshes were utilized for execution of the simulation. The simulations are resolved with a very fine mesh for all the cases (e.g. with 670000 cells for 3D cases) to predict the most accurate data. The schematic view of the studied grids is depicted in Fig. 4. 


\section{METHODOLOGY}

Solver type was chosen as Density-Based because fluid is compressible also high-speed flow. Different pressure inlets were examined i.e. $0.4,1,4$ and 8 atm. The corresponding pressure outlets are then $0.2,0.5,2$ and 4 atm. Note that, total pressure should be considered since total pressure is summation of static pressure and dynamic pressure and may be calculated as (4).

$$
\text { Total pressure }=p+\frac{\rho}{2} V^{2}
$$

The studied fluid is air, which must be defined as ideal gas; due to the supersonic flow density, that is not constant during passing through the nozzle. Pressure-based solver is for low-speed incompressible flows, while density-based is mainly used for high-speed compressible flows. Both are now applicable to a broad range of flows (from incompressible to highly compressible), but the origins of the density-based formulation may give it an accuracy (i.e. shock resolution) advantage over the pressure-based solver for high-speed compressible flows [21]-[23].

There are two most typical turbulence models used in CFD simulations, $k-\varepsilon$ model $\& \mathrm{k}-\omega$ model. Both models are currently used for CFD. Sometimes, these two models have sizeable numerical differences. In most cases the difference is in convergence time and the number of iterations [24]. $k-\varepsilon$ model is more feasible for fully turbulent flows. The model performs poorly for complex flows involving high pressure gradient, separation, and strong streamline curvature. The most significant weakness is lack of sensitivity to adverse pressure gradients. Basically, this model is suitable for initial iterations, initial screening of alternative designs, and parametric studies [25]. $k$ - $\omega$ model allows for a more accurate near wall treatment with an automatic switch from a wall function to a low-Reynolds number formulation based on grid spacing. This model performs significantly better for complex boundary layer flows under adverse pressure gradient conditions. $k-\omega$ has significant advantages in numerical stability. This model underestimates the amount of separation for severe adverse pressure gradient flows [26].

However, based on the experience on the current case in Fluent, results for temperature are less sensitive to model choice and for velocity seem indifferent. Pressure results seem highly sensitive to both the model choice and the mesh. Two models are very different and it should be no surprise that some differences in consequences can be obtained from each model (this should be considered the norm rather than unusual).

\section{RESULTS AND DISCUSSION}

The pressure ratio over the nozzle and the temperature are 0.5 and $300 \mathrm{~K}$, respectively. The assumption is, air flows from left hand side in $1 \mathrm{~atm}(101325 \mathrm{~Pa})$ then exits from right hand side after the nozzle in $0.5 \mathrm{~atm}(50662.5 \mathrm{~Pa})$. The pressure ratio variation over the nozzle centerline gathered from the measurement is presented in TABLE, where $X(\mathrm{~m})$ and $X_{\text {throat }}$ (m) are instantaneous distance from the inlet and coordination of throat, respectively. While, $p_{t, \text { in }}(\mathrm{N} / \mathrm{m} 2(\mathrm{~Pa}))$ is inlet pressure.
The cases are not performing under low Reynolds number; hence, $k-\varepsilon$ model has been mostly used as the viscous model for all the present CFD calculations. However, other viscous models like $k-\omega$ were also examined and it was practically discovered that obtained results for temperature are less sensitive to model choice. However, the results for pressure were very sensitive to model choice.

TABLE I: MEASUREMENT DATA

\begin{tabular}{cc|cc}
\hline \hline$X / X_{\text {throat }}$ & $p / p_{t, \text { in }}$ & $X / X_{\text {throat }}$ & $p / p_{t, \text { in }}$ \\
\hline 0.33 & 0.958 & 1.413 & 0.438 \\
0.44 & 0.942 & 1.456 & 0.447 \\
0.55 & 0.927 & 1.5 & 0.451 \\
0.659 & 0.899 & 1.543 & 0.455 \\
0.769 & 0.849 & 1.586 & 0.454 \\
0.89 & 0.67 & 1.629 & 0.456 \\
1 & 0.368 & 1.672 & 0.457 \\
1.111 & 0.278 & 1.715 & 0.457 \\
1.154 & 0.283 & 1.758 & 0.459 \\
1.198 & 0.292 & 1.802 & 0.461 \\
1.241 & 0.282 & 1.845 & 0.464 \\
1.284 & 0.275 & 1.888 & 0.468 \\
1.327 & 0.281 & 1.931 & 0.469 \\
1.37 & 0.413 & 1.974 & 0.462 \\
\hline \hline
\end{tabular}

Having above table, comparison of measured data and the results of the simulation would be possible to justify measured data. The effects of the location of fluid flow over the nozzle centerline were conducted by drawing plot of $p / p_{t, i n}$ versus $X / X_{\text {throat }}$. Since the outcomes of simulations are drawn in form of $\phi$ vs. $x$ (where $\phi$ is an arbitrary parameter), they should be converted in the basis of the Eulerian specification of the flow field, knowing that $x_{\text {throat }}=0.05779 \mathrm{~m}$.

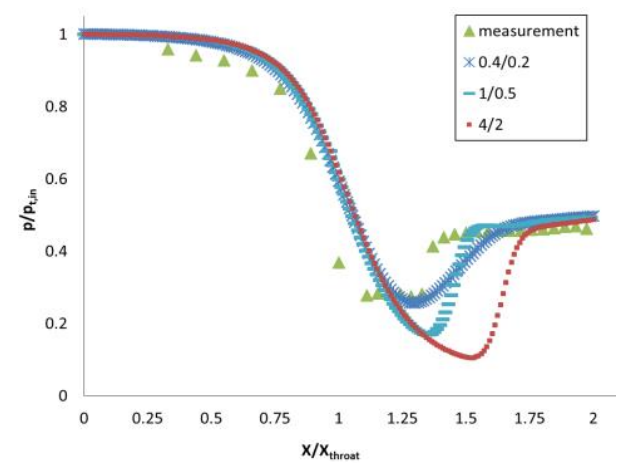

Fig. 5. Pressure along the nozzle centerline in 2D axisymmetric grid.

First of all an axisymmetric grid was examined. Pressure ratio diagram over the nozzle centerline is presented in Fig. 5. Different initial values are studied in this diagram but the pressure ratio over the nozzle is kept the same $\left(p_{\text {in }} / p_{\text {out }}=2\right)$.

Three different initial values show that lower inlet and outlet pressure is more similar to measuremet data $\left(p / p_{t, \text { in }}=\right.$ $0.4 / 0.2$ ) but shock effect is not as tangible as greater input pressure values in this case. Shock wave can be seen in greater inlet and outlet pressure, so that $p / p_{t, \text { in }}=4 / 2$ indicates that shock wave is happening in the span of 1.55-1.7. Based on the measurement data shock wave is occurring in the span of 1.35-1.4. The difference is caused by either inaccuracy in measurement or neglecting of some real phenomena like friction, or even both. 


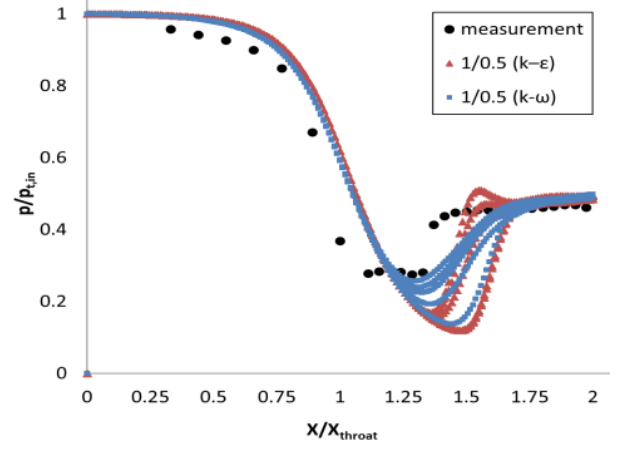

Fig. 6. Comparison of pressure over the nozzle centerline in k- $\varepsilon$ \& k- $\omega$ models.

Fig. 6 is exhibiting the distinction between $k-\varepsilon$ and $k-\omega$ model. Both models have identical initial value and set up whereas they obviously, have different pressure behavior in different time steps - due to the fact that their respective residuals never meet complete convergence - as a consequence of geometry after nozzle where supersonic flow and high Reynolds number have a key role in relatively distinct results of turbulence models as mentioned earlier. Pressure fluctuation over the nozzle will be eliminated through averaging method otherwise adverse outcomes are derived from the calculation.

However, pressure and the quality of shock wave are varying through the nozzle sequentially in a range, in other words nozzle will receive different shock waves in different iterations as it is clear in Fig. 6. Shock wave is more obvious in $\mathrm{k}-\varepsilon$ model, than $\mathrm{k}-\omega$ model, and with the higher trend to the lower pressure after nozzle but nevertheless it happens later than the measured case (and also $k$ - $\omega$ model).

In the further part of the work, a 2D complete (planner) case with the same properties as those of the previous axisymmetric grid was examined. As it is quite clear from the previous diagrams, the results of axisymmetric simulation do not exactly fit to the measurement data; consequently, a new geometry is required to be examined. To this end, planner (complete) grid is taken into account as the second case for CFD calculations. Boundary mesh is employed to critical boundaries; also the centerline preferably should have very fine mesh. Finally, defining the same model and properties as previous case, the simulation of the $2 \mathrm{D}$ complete (planner) case was accomplished.

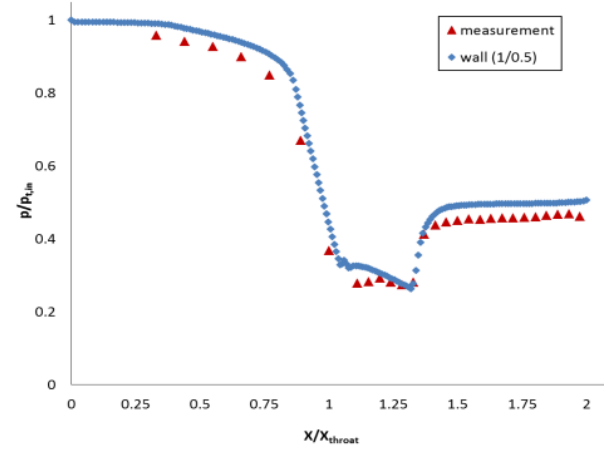

Fig. 7. Comparison of the measurement data and 2D complete (planner) simulation over the nozzle wall.

Same as axisymmetric case, the grid was examined in several initial values but the same pressure ratio over the nozzle. Comparison once again demonstrates that there is still difference between measurement data and data obtained from the CFD simulation cases. Fig. 7 represents the comparison of the measurement and the simulations data.

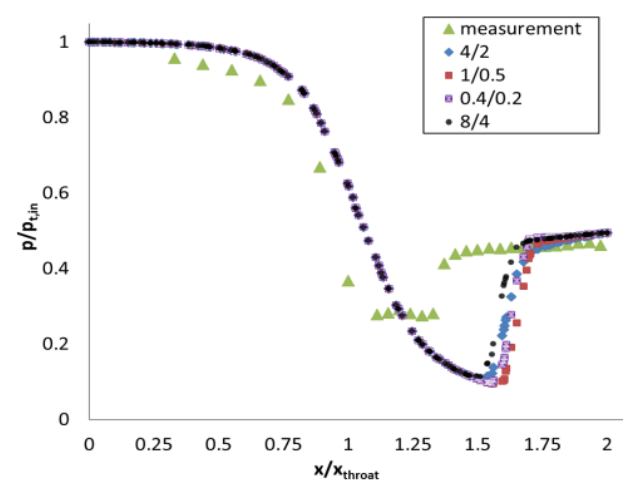

Fig. 8. Pressure in 3D case over the nozzle centerline.

A 3D model is also examined in which, outputs were very much more time consuming but very useful since determine correct result and verify some of the previous results. In other words, not only pressure over the nozzle is almost the same in all pressure values (diagram of pressure ratio over the nozzle centerline is almost same in all $P_{\text {in }} / P_{\text {out }}$ e.g. $1 / 0.5,8 / 4,4 / 2$ and $0.4 / 0.2$ in Fig. 8) but also other parameters are varying in the same way over the nozzle. As it has been mentioned, $k-\omega$ model is for low Reynolds numbers. Therefore, the result of $k-\omega$ model is neglected in this case.

In fact, CFD result is not strikingly varying in two discussed turbulence models. However, pressure change over the nozzle centerline in different pressure values is illustrated in Fig. 5. In all studied cases in 3D modeling shock wave is happening suddenly in a rage from 1.5 to 1.65 . The inlet and outlet pressure are determined in the basis of initial values, so that they are matching for the measurement data. The trend is roughly the same also the shock wave behavior is fairly acceptable (sudden pressure increase) and the difference is where shock wave occurs like in the most of $2 \mathrm{D}$ modeling cases.

For simplification, a plane is located at the center of the nozzle to monitor nozzle centerline. Fig. 9 illustrates the defined walls and center plane, which are further utilized for monitoring the data of centerline.

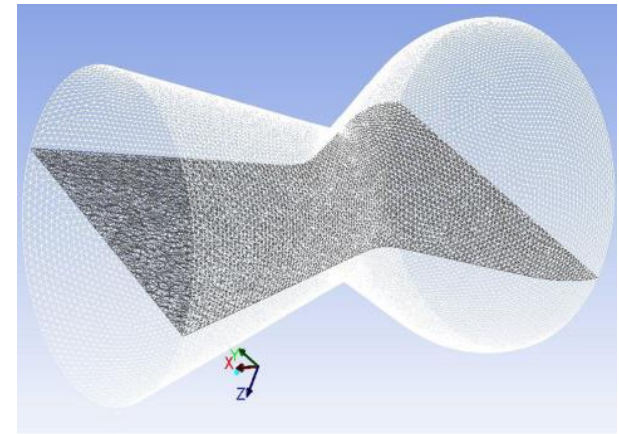

Fig. 9. 3D wall and center plane grid.

Results of CFD simulations are shown for pressure, velocity, temperature and turbulent kinetic energy when $P_{i n}$ is 4 and $P_{\text {out }}$ is 2 . Noteworthy that $P_{\text {in }}$ and $P_{\text {out }}$ stand for pressure at inlet and outlet of nozzle, respectively. 
It should be noted that profiles for $k-\varepsilon$ model and $k-\omega$ models were roughly identical, but since $k-\varepsilon$ model has more accuracy for supersonic converging-diverging nozzle, then $k$ - $\varepsilon$ model has been chosen for the illustration of graphical results.

Fig. 10 would certify previous pressure diagrams since blue area, which is a sudden mutation in pressure magnitude, is attributed to shock wave appearance. As shown in Fig. 11 velocity magnitude of flow over the nozzle is maximum (red area), where the pressure is minimum (blue area of the contour shown in Fig. 10). Flow after throat becomes supersonic till shock wave, so that suddenly become subsonic once again after shock and remains pretty steady until the outlet.

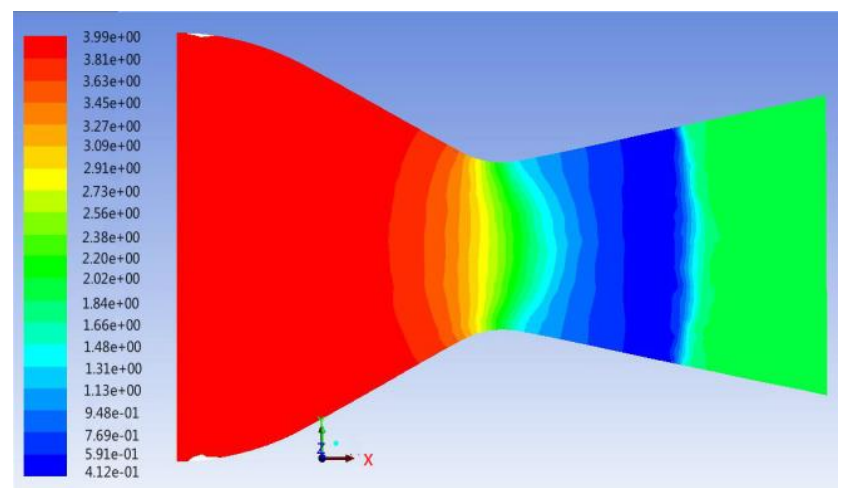

Fig. 10. Contour of static pressure.

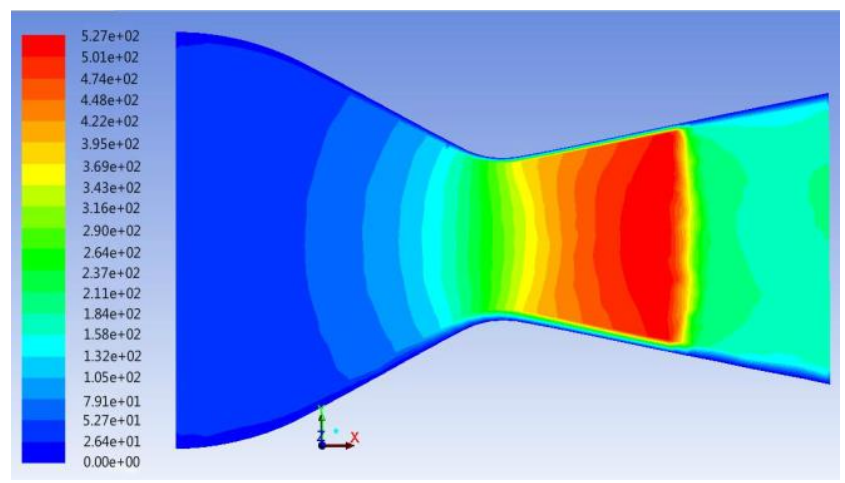

Fig. 11. Contour of velocity magnitude.

As illustrated in Fig. 12 temperature is minimum (blue area of the contour) while shock is occurring then immediately after shock start to grow with a sudden mutation. Fig. 13 also depicts the effect of turbulence in the nozzle since after shock turbulent kinetic energy has the highest value where the pressure has the lowest magnitude. Intensity of turbulence is bigger in the centerline than the wall.

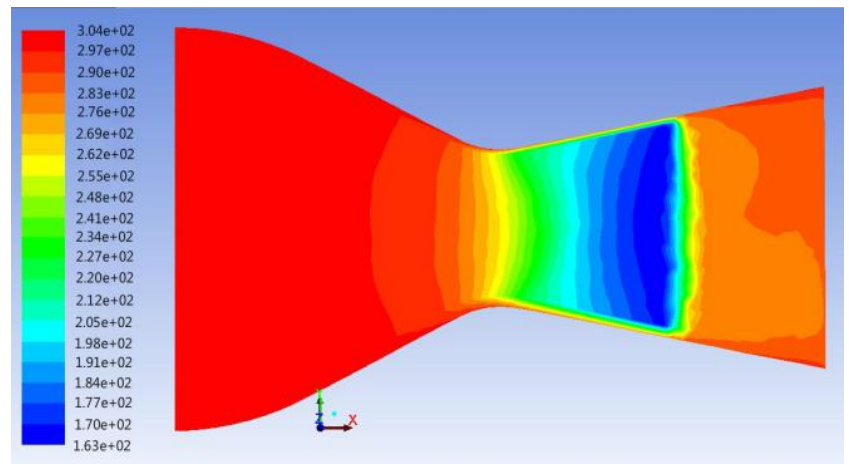

Fig. 12. Contour of static temperature.

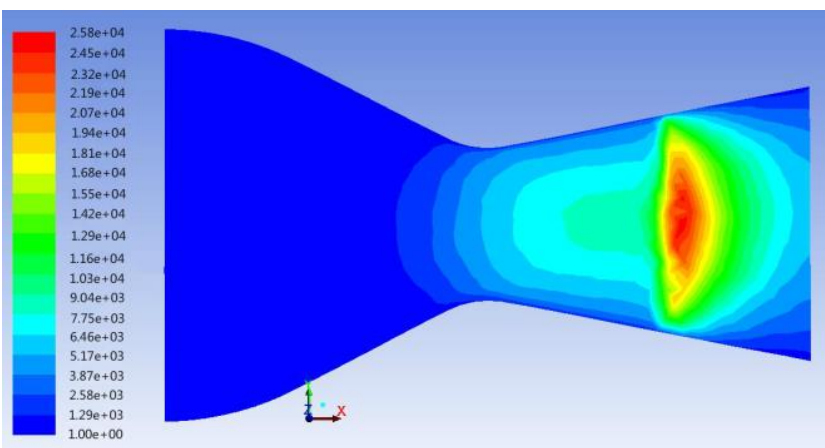

Fig. 13. Contour of turbulent kinetic energy.

Also contours for Mach number and density were drawn. Unsurprisingly, Mach number is 1 at the throat and increases slightly further until shock occurs, at where Mach number reaches around 2. On the other hand, based on the contour of density, it is discovered that the trend of density change is closely similar to that of pressure change. Moreover, analysis of the contour of wall shear stress demonstrates that the maximum shear stress happens at the throat, which is completely reasonable, and it decreases gradually through the diverging part of the nozzle toward the outlet.

\section{CONCLUSION}

In this geometry maximum Mach number is in the same place of minimum pressure in two turbulence models. This converging-diverging geometry does not let Mach number to reach more than this number. Since the velocity after the nozzle is supersonic, Mach number should be more than 1 after throat whereas Mach number is 1 in throat in both models but $k-\varepsilon$ model receives higher average values of Mach number in contrast to $\mathrm{k}-\omega$ model. It has been noticed that pressure in the throat and backpressure has to be identical (or very close). Green areas after shock and throat in Fig. 10 have the same Mach number value. As expected, the computational modeling outcomes are not completely matching for measured data. The neglect of real phenomena in CFD such as material property variation (specific heat capacity, thermal conductivity, viscosity), slip factor, wall friction etc. can be one reason. Also, selection of proper solver for each case (solution methods) is able to improve the outcomes and results.

Moreover, inaccuracy in measurements has a key role in final results as well. As author's opinion, for completion of computational modeling of a nozzle, pressure ratio is necessary but not sufficient. Having inlet (or outlet) pressure is essential to get reasonable result otherwise the result of CFD would be different for two considered case with inlet pressure of 4 atm and 1 atm although they have same pressure ratio.

Last but not least, CFD calculations might have different outcomes with different schemes and initial values such as grid, application, turbulence model, method, parameter magnitude and so on. Thus one can obtain true result in a single scheme while another one get different result through other scheme which could be also correct. In other words, CFD result of one case may not be the spitting image of the same case through other methods as expected. 


\section{ACKNOWLEDGMENT}

Authors acknowledge the support by the Academy of Finland through its COMP Center of Excellence grant (project no. 251748), and the EXPECTS project within the Aalto Energy Efficiency program.

\section{REFERENCES}

[1] T. Benson. (2013, July 25). Nozzle design, converging-diverging (CD) nozzle. National Aeronautics and space administration. [Online]. Available:https://www.grc.nasa.gov/www/k-12/airplane/nozzled.html.

[2] R. Boyanapalli et al., "Analysis of composite De-Laval nozzle suitable for rocket applications "International Journal of Innovative Technology and Exploring Engineering, vol. 2, pp. 336-344, 2013.

[3] T. W. Simpson et al., "Comparison of response surface and kriging models for multidisciplinary design optimization," American Institute of Aeronautics and Astronautics, vol. 98, pp. 1-16, 1998.

[4] J. J. Korte et al., "Multidisciplinary approach to linear aerospike nozzle design," Journal of Propulsion and Power, vol. 17, pp. 93-98.

[5] D. Foque et al., "Effects of nozzle type and spray angle on spray deposition in ivy pot plants," Pest Manag Sci, vol. 67, pp. 199-208.

[6] W. David and O. John, "SCORES - Web-based rocket propulsion analysis for space transportation system design," in Proc. 35th Joint Propulsion Conference and Exhibit, 1999.

[7] N. Zeoli et al., "CFD modeling of primary breakup during metal powder atomization," Chemical Engineering Science, vol. 66, pp. 6498-6504, 2011.

[8] A. J. Hewitt, "The importance of nozzle selection and droplet size control in spray application," in Proc. North American Conference on Pesticide Spray Drift Management, Portland, USA, 1998, pp. 75-85.

[9] T. Stevens et al., Steam Turbine Engineering, MacMillan, 1906.

[10] W. J. Devenport. (March 1 ${ }^{\text {st }}, 2001$ ). Nozzle applet. Applet. [Online]. Available:http://www.engapplets.vt.edu/fluids/CDnozzle/cdinfo.html.

[11] M. Darbandi and E. Roohi, "Study of subsonic-supersonic gas flow through micro/nanoscale nozzles using unstructured DSMC solver," Microfluidics and Nanofluidics, vol. 10, pp. 321-335, 2011.

[12] M. Einian. (2008). Compressible flow. CFD lab. University of Saskatchewan. [Online]. Available: http://www.engr.usask.ca/classes/ME/418/notes/T2-2008.ppt.

[13] W. Devenport, R. Kapania, K. Rojiani, and K. Singh. (2012). Java applets for engineering education. Virginia Polytechnic Institute and State University. National Science Foundation. [Online]. Available: http://www.engapplets.vt.edu/fluids/CDnozzle/cdinfo.html.

[14] S. Fielding, "The basic equations of fluid dynamics," in Laminar Boundary Layer Theory, Durham University, 2013, pp. 1-7.

[15] S. Fielding, "Further equations of fluid dynamics," in Laminar Boundary Layer Theory, Durham University, 2013, pp. 37-44.

[16] R. L. Bayt et al., "Viscous effects in supersonic mems-fabricated micronozzles," in Proc. the 3rd ASME Microfluids Symposium, 1998.

[17] G. S. E. Antipas et al., "Microstructural characterisation of Al-Hf and Al-Li-Hf spray deposits," Materials Characterization, vol. 62, pp. 402-408, 2011

[18] N. M. Khattab and M. H. Barakat, "Modeling the design and performance characteristics of solar steam-jet cooling for comfort air conditioning," Solar Energy, vol. 73, pp. 257-267, 2002.

[19] S. R. Turns, Thermodynamics Concepts and Applications, Cambridge University Press, 2006

[20] J. M. Cardemil and S. Colle, "A general model for evaluation of vapor ejectors performance for application in refrigeration," Energy Conversion and Management, vol. 64, pp. 79-86, 2012.

[21] Á. Veress and J. Rohács. (2011). Application of Finite Volume Method in Fluid Dynamics and Inverse Design based Optimization. Budapest University of Technology and Economics. [Online]. Available: www.intechopen.com/download/pdf/33899.

[22] H. Zheng et al., "Computational fluid dynamics simulation of the supersonic steam ejector. Part 1: Comparative study of different equations of state," J. of Mech. Eng. Sci., vol. 226, pp. 709-714, 2012.

[23] Z. Chen and A. Przekwas, "A coupled pressure-based computational method for incompressible/compressible flows," Journal of Computational Physics, vol. 229, pp. 9150-9165, 2010.

[24] F. Menter and Y. Egorov, "The scale-adaptive simulation method for unsteady turbulent flow predictions. Part 1: Theory and mode description," Flow, Turbulence and Combustion, vol. 85, pp. 113-138.

[25] G. Mylavarapu et al., "Validation of computational fluid dynamics methodology used for human upper airway flow simulations," Journal of Biomechanics, vol. 42, pp. 1553-1559, 2009.
[26] F. R. Menter, "Performance of popular turbulence models for attached and separated adverse pressure-gradient flows," Aiaa Journal, vol. 30, pp. 2066-2072, Aug. 1992

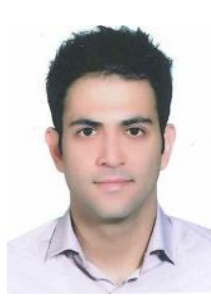

Omid Joneydi Shariatzadeh was born in Tehran, Iran, on September 21, 1984. He received his BSc. degree in mechanical engineering from Zanjan University, Zanjan, Iran in 2008, MSc. degree in energy technology from Lappeenranta University of Technology, Lappeenranta, Finland in 2013.

$\mathrm{He}$ is currently a research assistant at laboratory of thermodynamics, Lappeenranta University of Technology. His research interests are heat and mass transfer, CFD, multiphase flows, modeling of energy systems and thermal science.

He has collaborated in more than 6 ISI articles, 3 conference papers. He has also worked as the head of mechanics and CAD division, in the field of design and modeling of energy systems at ASA Consulting Engineers Company, Tehran, Iran.

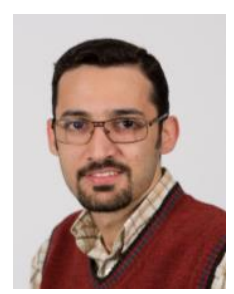

Afshin Abrishamkar was born in Kobe, Japan. He received his B.Sc. degree in chemical engineering from Isfahan University of Technology, Iran in 2011 Subsequently he obtained his M.Sc. degree with distinction in chemical and process engineering at Lappeenranta University of Technology, in 2014.

During his undergraduate studies, he accomplished a technical internship at Centro Federal de Educação Tecnológica de Minas Gerais, Brazil working on optimization of biodiesel production. Moreover, he conducted his master's thesis, as an invited visiting student, at Swiss Federal Institute of Technology Zurich, Switzerland in 2013. His master's thesis was mainly involved in large scale synthesis of microdroplets in microfluidics. He is currently a researcher at Department of Chemical Technology at Lappeenranta University of Technology. So far, he has published more than 8 scientific papers in peer reviewed journals and international conferences. Furthermore, in 2010, he co-established a designing/consulting company in Iran, at where he remained for two years as the director of finance $\&$ administration affairs (maintaining the position of Vice Chairperson of the Board) for two years. Since then, he has been remotely serving at same company as the director of planning and international relations. His research interests lie in various chemical fields such as: microfluidics, process engineering, computational modeling of processes, and reaction engineering, among others.

Mr. Abrishamkar has been selected as a scientific reviewer for ECOS 2014. In 2013, he was awarded a "research fellowship" by Lappeenranta University of Technology, Finland to conduct his master's thesis abroad. In the same year, he received "Excellence in Studies Award" from The Research Foundation of Lappeenranta University of Technology, Finland based on his outstanding records during his M.Sc.

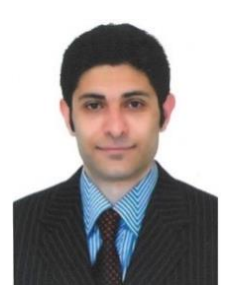

Aliakbar Joneidi Jafari was born in Tehran, Iran. He received his BSc. degree in mechanical engineering from University of Tehran, Iran in 2010. Then, he has pursued his MSc. studies in Lappeenranta University of Technology, in environmental energy technology field, while his master thesis is being done in Aalto University, Espoo, Finland.

He has had part time work experiences for a few years in industry especially in different car manufacturing companies and their linked industries as designer, manufacturer and trainee. He is currently a research assistant working in Applied Thermodynamics Lab of Energy and Applied Physics Departments in Aalto University, Espoo, Finland. Moreover, he patented a few inventions pertaining to the vehicles, designed and manufactured renewable energy apparatuses such as solar cooker and flat plate collector. Furthermore, he has had a great experience in product design and development of a whole traditional bakery system to be renovated with automatic processes. In addition, he has published some articles on heat transfer and fluid mechanics especially in nanofluid implementations. His research interests are utilization of renewable energies chiefly in buildings, entrepreneurship in energy industry mainly in intelligent buildings which can augment social value and finally, assessment of energy consumers to find solutions to increase their energy efficiency.

Mr. Joneidi Jafari is a member of EXPECTS one of the 9 consortia of AEF (Aalto Energy Efficiency program). He has been awarded the scholarship for the whole years of bachelor and master studies. 
